\title{
LGBTQ + health: a failure of medical education
}

\author{
Michael A. Gisondi ${ }^{1}$ [ $\cdot$ Blair Bigham ${ }^{2}$
}

Received: 9 July 2021 / Accepted: 19 July 2021

(C) The Author(s), under exclusive licence to Canadian Association of Emergency Physicians (CAEP)/ Association Canadienne de Médecine d'Urgence (ACMU) 2021

Keywords Lesbian · Gay · Transgender · Queer · Medical education · LGBTQ+ health

\section{The historical context of the training gap}

Lien, Vujcic, and Ng identify a contemporary failure of medical education in their article, "Attitudes, Behaviour, and Comfort of Canadian Emergency Medicine Residents and Physicians in Caring for 2SLGBTQI + Patients". The authors surveyed faculty and resident physicians in emergency medicine about their comfort, current knowledge, and desire to learn to care for LGBTQ + patients. Almost all respondents agreed that queer patients deserve quality healthcare equal to that of non-queer patients. Most of those surveyed were never trained to address the unique health needs of LGBTQ + patients and, not surprisingly, many of these physicians were uncomfortable caring for this patient population. Importantly though, most respondents wanted to improve their knowledge of the care of queer patients.

The findings of this study aren't simply explained by discordant provider attitudes, behaviors, or comforts, as the title of the article might have you believe. Instead, at the root of the problem, these findings stem from years and years of failing to teach physicians about queer people. Specifically, these findings correspond to the longstanding pathologization of queerness during the AIDS crisis of the 1980s and 1990s, and the dearth of medical school curriculum devoted to LGBTQ + patients ever since [1]. In fact, LGBTQ + health content has been presented only in the context of HIV/ AIDS for decades in most medical schools, at the expense of many other queer health topics. The historical failure to train

Michael A. Gisondi

mgisondi@stanford.edu

1 Department of Emergency Medicine, Stanford School of Medicine, 900 Welch Road, Suite 350, Stanford, Palo Alto, CA, USA

2 Critical Care Medicine, Stanford School of Medicine, Stanford, CA, USA physicians meant that those surveyed in this study didn't learn about LGBTQ + health because their faculty members before them didn't learn about LGBTQ + health. Today, at a time when queer people are now widely accepted in society and physicians want to learn about LGBTQ + health, there are very few providers trained to teach the content. There remains a massive knowledge gap among the majority of practicing physicians when it comes to queer health, and a lack of people competent to teach what needs to be taught.

It is worth noting several disturbing findings in this study that relate to the longstanding training gap. The authors found that a whopping $33 \%$ of physicians are uncomfortable caring for queer patients, despite being overwhelmingly comfortable working alongside LGBTQ + staff and agreeing that LGBTQ + patients should get equal healthcare. Why? Why do $21 \%$ of physicians maintain poor eye contact with their queer patients? Why do $45 \%$ of respondents avoid asking about sexual behaviors in a patient population that is defined in large part by their sexuality? And most concerning, why are emergency medicine residents significantly less likely to perform procedures on LGBTQ + patients? The simple answer: a lack of training.

After pathologizing queer people in medicine for so long, is it really all that surprising that physicians exhibit poor eye contact, inadequate health histories, and avoidance of bedside care? If those are the current practices of emergency physicians, then medical education has certainly failed queer patients.

\section{Closing the training gap will improve patient care}

Trainees in this study wanted to learn more about the care of LGBTQ + patients, yet faculty members are unprepared to train them $[2,3]$. Teaching queer health requires faculty development for even introductory content, as many 
clinician-educators have little knowledge of LGBTQ + health or how to teach it. We must uniformly train all faculty and students in the basics: LGBTQ+ vocabulary, social and behavioral determinants of queer health, creation of welcoming and inclusive health care spaces, legal issues for unmarried partners, medical prevention of HIV, care of transgender patients, and pelvic health in patients assigned female at birth.

We believe that all physicians, regardless of specialty, should be trained in these fundamentals of LGBTQ+ health. Such training would ensure more comfortable, respectful, and productive encounters for patients and providers. In emergency medicine, LGBTQ + health is similar to many other low-frequency, high-stakes conditions that require targeted provider education. Therefore, all emergency physicians should be adequately trained in the basics of LGBTQ+ health. Such knowledge will empower emergency physicians to confidentially and respectfully assess queer patients, manage their presenting conditions, and refer them to appropriate specialists for aftercare. We meet our training requirements for other unique patient populations, LGBTQ + health should be no different.

Some skeptics suggest that education alone cannot drive practice change. For education to work, there often needs to be corresponding pressure on the learner, such as regulatory mandates, normative influences, or financial incentives. With respect to LGBTQ + health content, all we have right now is education, as there are no practice mandates. But that could change. We could structurally incentivize and mandate training in many different ways. Regulators, colleges, medical schools, and hospitals could include LGBTQ + health training as a requirement for certification, licensure, and employment. And importantly, we could create engaging learning resources for busy physicians asked to meet additional training requirements. One example is Teaching $L G B T Q+$ Health, a free online CME course offered by Stanford University [4]. The course is comprehensive, covers the core principles of queer health, and is self-paced.

Beyond provider training, we could address gaps in patient care by designating some emergency departments as centres of excellence in LGBTQ + health, not unlike the way stroke, trauma, and sexual assault centres are designated.
This would conveniently mirror the specialization of LGBTQ + clinics, health centers, and community services. Such designations would require emergency physicians to be knowledgeable and competent in queer health.

Decades of physicians poorly trained in LGBTQ + health represent a persistent failure of the medical education system. Change begins with improved education... yours. Choose an appropriate resource and learn about your queer patients. Share the resource with members of your practice and colleagues outside your institution. Then teach a few trainees. End the cycle of inadequate training on LGBTQ + health. This is how the House of Medicine can catch up. It would be nice to see these changes happen soon... and a survey study with better results thereafter.

\section{Declarations}

Conflict of interest Michael A. Gisondi is the Course Director for Stanford's Teaching LGBTQ+ Health course mentioned in this article. He has no financial incentive to serve in this role and the number of course participants is not financially rewarded. Blair Bigham has no disclosures to report.

\section{References}

1. Florêncio J. AIDS: homophobic and moralistic images of $1980 \mathrm{~s}$ still haunt our view of HIV - that must change. The Conversation; 2018. https://theconversation.com/aids-homophobic-and-moral istic-images-of-1980s-still-haunt-our-view-of-hiv-that-mustchange-106580. Accessed 8 July 2021.

2. Hollenbach AD, Eckstrand KL, Dreger A, editors. Implementing curricular and institutional climate changes to improve health care for individuals who are LGBT, gender nonconforming, or born with DSD: a resource for medical educators. Association of American Medical Colleges; 2014. https://store.aamc.org/downl oadable/download/sample/sample_id/129/. Accessed 8 July 2021.

3. Obedin-Maliver J, Goldsmith ES, Stewart L, et al. Lesbian, gay, bisexual, and transgender-related content in undergraduate medical education. JAMA. 2011;306(9):971-7.

4. Gisondi MA, Keyes T, Bumgardner D, Zucker S. Teaching LGBTQ + Health: a faculty development course for health professions educators. Stanford Medicine; 2021. https://mededucati on.stanford.edu/courses/teaching-lgbtq-health/. Accessed 8 July 2021. 